\title{
Terquem theorem with the spherical helix strip
}

\author{
Filiz Ertem Kaya \\ Department of Mathematics, Faculty of Science and Arts, Nigde University, Nigde, Turkey
}

Email address:

fertem@nigde.edu.tr

\section{To cite this article:}

Filiz Ertem Kaya. Terquem Theorem with the Spherical Helix Strip. .Pure and Applied Mathematics Journal. Special Issue: Applications of Geometry.Vol. 4, No.1-2, 2015 pp.1-5.doi: 10.11648/j.pamj.s.2015040102.11

\begin{abstract}
The spherical helix and the strip are respectively proved firstly by Scofield and Sabuncuoglu and Hacisalihoglu. In this paper helix strip on sphere is investigated by using characteristics of spherical helix and strip. Firstly using strip after helixand finally spherical helix. So spherical helix strips are obtained. Furhermore Joachimsthal Theorem and Terquem Theorem are investigated when the strip and helix strips which lie on the sphere and given a characterization about spherical helix strips.
\end{abstract}

Keywords: Helix, Strip, Spherical Helix Strip, 2010 Mathematics Subject Classification. 53A04, 53A05

\section{Introduction}

Struikand Blaschke investigated that if a helix has constant curvature, then its projection on a plane perpendicular to its axis is a plane curve of constant curvature, hence circle and the spherical helix projects on a plane perpendicular to its axis in an arc of an epicycloid. Sabuncuoglu and Hacisalihoglu proved higher curvatures of curve-surfave pair (we use the strip as curve-surface pair) andKeles showed some relations of curve-surface pair on two different surfaces that we know these asJoachimsthaltheorems .

In 3-dimensional Euclidean Space, a regular curve is described by its curvatures $k_{1}$ and $k_{2}$ and also a strip is descibed by its curvatures $k_{n}, k_{g}$ and $t_{r}$. The relations between the curvatures of a strip and the curvatures of the curve can be seen in many differential books and papers. We know that a regular curve is called a general helix if its first and second curvatures $k_{1}$ and $k_{2}$ are not constant, but $\frac{k_{1}}{k_{2}}$ is constant ([2],[8]). Also if a helix lie on a sphere,it is called a spherical helix.

The spherical helix provides being a helix condition and the sphericity condition by using the curvatures of helix $k_{1}$ and $k_{2}$. Also Joachimsthal Theorem and Terquem Theorem (known as one of Joachimsthal Theorems but we call Terquem Theorem for easiness) are proved when the helix strip that lies on sphere.

\section{The Curve-Surface Pair (Strip)}

Definition :

Let $M$ and $\alpha$ be a surface in $E^{3}$ and a curve in $M \subset E^{3}$. We define a surface element of $M$ is the part of a tangent plane at the neighbour of the point. The locus of these surface element along the curve $\alpha$ is called a curve-surface pair and is shown as $(\alpha, M)$.

Definition 2:

Let $\{\vec{t}, \vec{n}, \vec{b}\}$ and $\{\overrightarrow{\boldsymbol{\xi}}, \vec{\eta}, \vec{\zeta}\}$ be the curve and curvesurface pair's vector fields. The curve-surface pair's tangent vector field, normal vector field and binormal vector field is given

$$
\text { by } \vec{t}=\vec{\xi}, \vec{\zeta}=\vec{N}(\vec{N}=\vec{n}) \text { and } \vec{\eta}=\vec{\zeta} \wedge \vec{\xi}([8,10,11]) \text {. }
$$

\subsection{Curvatures of the Curve-Surface Pair and Curvatures of the Curve}

Let $k_{n}=-b, k_{g}=c, t_{r}=a$ and $\{\overrightarrow{\boldsymbol{\xi}}, \overrightarrow{\boldsymbol{\eta}}, \vec{\zeta}\}$ be the normal curvature, the geodesic curvature, the geodesic torsion of the strip and the curve-surface pair's vector fieldson $\alpha[8,10,11]$.

Then we have 


$$
\begin{aligned}
& \vec{\xi}^{\prime}=c \vec{\eta}-b \vec{\zeta} \\
& \vec{\eta}^{\prime}=-c \vec{\xi}+a \vec{\zeta} \\
& \vec{\eta} \\
& \vec{\zeta}=b \vec{\xi}-a \vec{\eta} .
\end{aligned}
$$

We know that a curve $\alpha$ has two curvatures $\kappa$ and $\tau$. A curve has a strip and a strip has three curvatures $k_{n}, k_{g}$ and $t_{r}$.

Let $k_{n}, k_{g}$ and $t_{r}$ be the $-b, c$ anda. From last equations we have $\vec{\xi}=c \vec{\eta}-b \vec{\zeta}$. If we substitude $\vec{\xi}=\vec{t}$ in last equation, we obtain

$$
\vec{\xi}=\kappa_{n}
$$

and

$$
\begin{aligned}
& b=-\kappa \sin \phi \\
& c=\kappa \cos \phi
\end{aligned}
$$

$([8,10,11])$.

From last two equations we obtain,

$$
\kappa^{2}=b^{2}+c^{2} .
$$

This equation is a relation between the curvature $\kappa$ of a curve $\alpha$ and normal curvature and geodesic curvature of a curve-surface pair.

By using similar operations, we obtain a new equation as follows

$$
\tau=a+\frac{b^{\prime} c-b c^{\prime}}{b^{2}+c^{2}}
$$

([8,10,11]).

This equation is a relation between $\tau$ (torsion or second curvature of $\alpha$ and curvatures of a curve-surface pair that belongs to the curve $\alpha$ ).And also we can write

$$
a=\phi^{\prime}+\tau \text {. }
$$

The special case:

If $\phi$ is constant, then $\phi^{\prime}=0$. So the equation is $a=\tau$. That is, if the angle is constant, then torsion of the curvesurface pair is equal to torsion of the curve.

Definition:

Let $\alpha$ be a curve in $M \subset E^{3}$. If the geodesic curvature (torsion) of the curve $\alpha$ is equal to zero, then the curvesurface pair $(\alpha, M)$ is called a curvature curve-surface pair (strip) $([8,10,11])$.

\section{General Helix}

Definition:
Let $\alpha$ be a curve in $E^{3}$ and $V_{1}$ be the first Frenet vector field of $\alpha . U \in \chi\left(E^{3}\right)$ be a constant unit vector field.

If

$$
\left\langle V_{1}, U\right\rangle=\cos \phi \text { (constant) }
$$

$\alpha, \phi$ and $S p\{U\}$ are called a general helix, the slope and the slope axis $([1,2,6])$.

Definition:

A regular curveis called a general helix if its first and second curvatures $\kappa$ and $\tau$ are not constant but $\frac{\kappa}{\tau}$ is constant $([1,6])$.

Definition: A curve is called a general helix or cylindrical helix it its tangent makes a constant angle with a fixed line in space. A curve is a general helix if and only if the ratio $\frac{\kappa}{\tau}$ is constant $([5,9,12])$.

Definition: A helix is a curve in 3-dimensional space. The following parametrisation in Cartesian coordinates defines a helix, see [7].

$$
\begin{aligned}
& x(t)=\cos t \\
& y(t)=\sin t \\
& z(t)=t .
\end{aligned}
$$

As the parameter $t$ increases $(x(t), y(t), z(t))$ traces a right-handed helix of pitch $2 \pi$ and Radius 1 about the $z$ axis, in a right-handed coordinate system. In cylindrical coordinates $(r, \theta, h)$ the same helix is parametrised by

$$
\begin{aligned}
& r(t)=1, \\
& \theta(t)=t, \\
& h(t)=t .
\end{aligned}
$$

\section{Definition:}

If the curve $\alpha$ is a general helix, the ratio of the first curvature of the curve to the torsion of the curve must be the constant. The ratio $\frac{\tau}{\kappa}$ is called first harmonic curvature of the curve and is denoted by $H_{1}$ or $H$.

Theorem 3.1: A regular curve $\alpha \subset E^{3}$ is a general helix if and only if $H(s)=\frac{k_{1}}{k_{2}}=$ const for $\forall s \in I$, see [7].

Proof: $(\Rightarrow)$ Let $\alpha$ be a general helix. The slope axis of the curve $\alpha$ is showed $\operatorname{Sp}\{U\}$. Note that

$$
\left\langle\alpha^{\prime}(s), U\right\rangle=\cos \phi=\text { const } .
$$

If the Frenet Threshold is $V_{1}, V_{2}, V_{3}$ at the point $\alpha(s)$, then we have

$$
\left\langle V_{1}(s), U\right\rangle=\cos \phi
$$


If we take derivative of the both sides of the last equation, then we have

$$
\left\langle k_{1} V_{2}(s), U\right\rangle=0 \Rightarrow\left\langle V_{2}(s), U\right\rangle=0 .
$$

Hence

$$
U \in \operatorname{Sp}\left\{V_{1}(s), V_{3}(s)\right\}
$$

Therefore

$$
U=\cos \phi V_{1}(s)+\sin \phi V_{3}(s)
$$

$U$ is the linear combination of $V_{1}(s)$ and $V_{3}(s)$. By differentiating the equation $\left\langle V_{2}(s), U\right\rangle=0$, we obtain

$$
\begin{aligned}
& \left\langle-k_{1} V_{1}(s)+k_{2} V_{3}(s), U\right\rangle=0, \\
& -k_{1}(s)\left\langle V_{1}(s), U\right\rangle+k_{2}(s)\left\langle V_{3}(s), U\right\rangle=0, \\
& -k_{1}(s) \cos \phi+-k_{2}(s) \sin \phi=0 .
\end{aligned}
$$

By using the last equation, we see that

$$
H=\text { const. }
$$

$(\Leftarrow)$ Let $H(s)$ be constant for $\forall s \in I$, and $\lambda=\tan \phi$, then we obtain

$$
U=\cos \phi V_{1}(s)+\sin \phi V_{3}(s)
$$

1 If $U$ is a constant vector, then we have

$$
D_{\alpha} U=\left(k_{1}(s) \cos \phi-\sin \phi k_{2}(s)\right) V_{2}(s)
$$

By substituting $H(s)=\tan \phi$ is in the last equation, we see that

$$
k_{1}(s) \cos \phi-k_{2} \sin \phi=0
$$

and so

$$
U=\text { const } \text {. }
$$

2 If $\alpha$ is an inclined curve with the slope axis $\operatorname{Sp}\{U\}$, then

$$
\begin{aligned}
\left\langle\alpha^{\prime}(s), U\right\rangle & =\left\langle V_{1}(s), \cos \phi V_{1}(s)+\sin \phi V_{3}(s)\right\rangle \\
& =\cos \phi\left\langle V_{1}(s), V_{1}(s)\right\rangle+\sin \phi\left\langle V_{1}(s), V_{3}(s)\right\rangle
\end{aligned}
$$

and we obtain

$$
\left\langle\alpha^{\prime}(s), U\right\rangle=\cos \phi=\text { const }
$$

([7]).

Definition:

Let $S^{2}$ and $\alpha$ be a sphere in $E^{3}$ and a helix that lies on the sphere $S^{2}$. The curve $\alpha$ is called a spherical helix which lie on the sphere[12].

\section{Definition:}

Let $\alpha$ be a helix in $M \subset E^{3}$. We define a surface element of $M$ is the part of a tangent plane at the neighbour of the point of the helix that lie on $M$. Instead of the geometric plane of these surface elements along the helix $\alpha$ which lie sphere $M$ is called a helix strip.

\section{Definition:}

Let $S^{2}$ be a sphere and and $\alpha$ a helix which lie on $S^{2}$ in $E^{3}$. We define a surface element $S^{2}$ is the part of a tangent plane at the neighbour of the point of the helix that lie on $S^{2}$. The locus of these surface elements along the helix $\alpha$ which lie on the sphere $S^{2}$ is called spherical helix strip.

Theorem 3.2 ${ }^{[10]}$ : (Terquem Theorem) Let $M_{1}$ and $M_{2}$ be the different surfaces in $E^{3}$ and $\alpha$ be a curve but not a planar curve and $\beta$ be a curve on $M_{2}$.

i The points of the curves $\alpha$ and $\beta$ corresponds to each other $1: 1$ on a plane $\varepsilon$ which rolls on the $M_{1}$ and $M_{2}$, such that the distance is constant between corresponding points.

ii $\left(\alpha, M_{1}\right)$ is a curvature strip.

iii $\left(\beta, M_{2}\right)$ is a curvature strip.

Proof: Claim:Two of the three lemmas give the third $([5,10])$. It is obviously from the doctoral dissertation by Keles.

By applying the similar way in proof of the Theorem 3.2 in $[5,10]$ to the strip of the spherical helix strip, we give the following theorem.

Theorem 3.3: Let $S^{2}$ be a sphere and and $M$ be a surface in $E^{3}$. Let the tangent planes of the surface $M$ that along the curve $\beta$ be the tangent planes of the sphere $S^{2}$ along the helix curve $\alpha$ at the same time. In this case, if the curvesurface pair $(\beta, M)$ is a curvature strip, the curve $\beta$ is a helix and helix strip.

Proof:

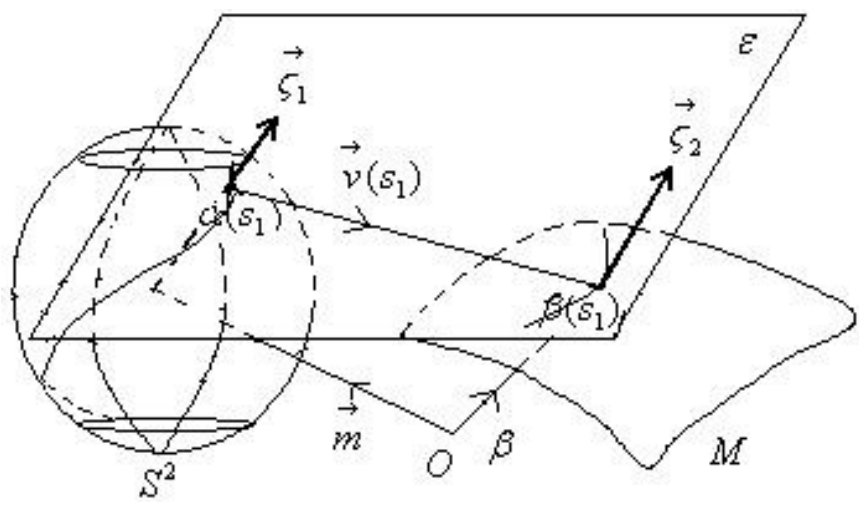

Figure.Spherical helix $S^{2}$ and Surface $M$

We use all of the proof of the Terquem Theorem of doctoral thesis of Keles, but only different is we should prove the helix strip.If the curve $\alpha$ is a helix on $S^{2}$, then it 
provides $\frac{\kappa_{1}}{\tau_{1}}$ is constant. We have to show that $\beta$ is a helix strip on $M$, that is, $\frac{\kappa_{2}}{\tau_{2}}$ is constant.

By the Figure, we have

$$
\beta\left(s_{1}\right)=\alpha\left(s_{1}\right)+\lambda\left(s_{1}\right) \vec{v}\left(s_{1}\right)
$$

where

$$
\alpha\left(s_{1}\right)=\vec{m}+r \zeta_{1}\left(s_{1}\right)
$$

By differentiating both side of (3), we see that

$$
\vec{\xi}_{1}=\frac{d \alpha_{1}}{d s_{1}}=r \frac{d \zeta_{1}}{d s_{1}}
$$

By (1),

$$
\begin{aligned}
& \vec{\xi}_{1}=r\left(b_{1} \xi_{1}-a_{1} \eta_{1}\right), \\
& \qquad \frac{d \beta}{d s_{1}}=\frac{d \vec{m}}{d s_{1}}+\frac{d \vec{\zeta}_{1}}{d s_{1}}+\frac{d \lambda}{d s_{1}}\left(\cos \phi \vec{\xi}_{1}+\sin \vec{\eta}_{1}\right)+\lambda\left(s_{1}\right) \frac{d\left(\cos \phi \vec{\xi}_{1}+\sin \phi \vec{\eta}_{1}\right)}{d s_{1}}
\end{aligned}
$$

Since the vector $\vec{m}$ and $\lambda$ are constant, we obtain the following equation

$$
\frac{d \beta}{d s_{1}}=\frac{d \vec{\zeta}_{1}}{d s_{1}}+\lambda\left(s_{1}\right) \frac{d\left(\cos \phi \vec{\xi}_{1}+\sin \phi \vec{\eta}_{1}\right)}{d s_{1}}
$$

or

$\left.\frac{d \beta}{d s_{1}}=\frac{d \vec{\zeta}_{1}}{d s_{1}}+\lambda\left(s_{1}\right)\left(-\frac{d \phi}{d s_{1}} \sin \phi \vec{\xi}+\cos \phi \frac{d \xi_{1}}{d s_{1}}\right)+\frac{d \phi}{d s_{1}} \cos \phi \vec{\eta}_{1}+\sin \phi \frac{d \vec{\eta}_{1}}{d s_{1}}\right)$.

By (1), we obtain

$\frac{d \beta}{d s_{1}}=\left[1-\lambda\left(\frac{d \varphi}{d s_{1}}+c_{1}\right) \sin \phi\right] \vec{\xi}_{1}+\lambda\left(\frac{d \phi}{d s_{1}}+c_{1}\right) \cos \phi \vec{\eta}_{1}-\lambda \cos \phi \vec{\zeta}_{1}$

Since the spherical helix and the surface $M$ have the same tangent plane along the curves $\alpha$ and $\beta$, we can write

$$
\left\langle\frac{d \beta}{d s_{1}}, \vec{\zeta}_{1}\right\rangle=0
$$

By substituting (6) at the last equation, we obtain $\cos \phi=0$. By using that equation in (6), we have

$$
\frac{d \beta}{d s_{1}}=\left(1 \pm \lambda c_{1}\right) \vec{\xi}_{1}
$$

If we calculate the second and third derivatives of the
We obtain $a_{1}=0$ and $b_{1}=1$.

$r$ is the radius of the sphere. We denote $r=1$. Since $\vec{m}$ is a position vector that goes to the center of the sphere, $\vec{m}$ is constant.

Since $\alpha_{1}=0,\left(\alpha, S^{2}\right)$ is a curvature strip. By the strips $\left(\alpha, S^{2}\right)$ and $(\beta, M)$ are curvature strips and by the Terquem Theorem, we see that $\lambda$ is non-zero constant. Let $\vec{v}\left(s_{1}\right)$ be a vector in $\operatorname{Sp}\left\{\xi_{1}, \eta_{1}\right\}$, and let $\phi$ be the angle between $\vec{\xi}_{1}$ and $\vec{v}\left(s_{1}\right)$. Then we write

$$
v\left(\vec{s}_{1}\right)=\cos \phi \vec{\xi}_{1}+\sin \phi \vec{\eta}
$$

By substituting (3) and (4) in (2), and differentiating both sides, we obtain (5). curve $\beta$, then we get

$$
\begin{gathered}
\frac{d^{2} \beta}{d s^{2}}=\mp \lambda c_{1}^{\prime} \vec{\xi}_{1}+\left(1 \mp \lambda c_{1}\right) c_{1} \vec{\eta}_{1}-\left(1 \mp \lambda c_{1}\right) \vec{\zeta}_{1}- \\
\frac{d^{3} \beta}{d s_{1}{ }^{3}}=\left[\mp \lambda c^{\prime \prime}-\left(1 \mp \lambda c_{1}\right) c_{1}^{2}-\left(1 \mp \lambda c_{1}\right)\right] \vec{\xi}_{1}+ \\
\left.\left[\mp \lambda c_{1} c_{1}^{\prime} \mp \lambda c_{1} c_{1}^{\prime}+\left(1 \mp \lambda c_{1}\right) c_{1}\right]\right] \vec{\eta}_{1}+\left(\mp \lambda c_{1}^{\prime} \mp \lambda c_{1}^{\prime}\right) \vec{\zeta}_{1}
\end{gathered}
$$

Since the same result is obtained by using other form of (7), we use the form $\frac{d \beta}{d s_{1}}=\left(1-\lambda c_{1}\right) \vec{\xi}_{1}$ of (7) at the rest of our proof. By differentiating both sides of (7), we obtain $\frac{d \beta}{d s_{1}}=\left(1-\lambda c_{1}\right) \vec{\xi}_{1}$ $\frac{d^{2} \beta}{d s_{1}^{2}}=-\lambda c_{1}^{\prime} \vec{\xi}_{1}+\left(1-\lambda c_{1}\right) c_{1} \vec{\eta}_{1}-\left(1-\lambda c_{1}\right) \vec{\zeta}_{1}$ $\frac{d^{3} \beta}{d s_{1}{ }^{3}}=\left[-\lambda c^{\prime \prime}-\left(1-\lambda c_{1}\right) c_{1}^{2}-\left(1-\lambda c_{1}\right)\right] \vec{\xi}_{1}+\left[3 \lambda c_{1} c_{1}^{\prime}+c_{1}^{\prime}\right] \vec{\eta}_{1}+\left(2 \lambda c_{1}^{\prime}\right) \vec{\zeta}_{1}$

By applying Gram-Schmidt to the $\left\{\beta^{\prime}, \beta^{\prime \prime}, \beta^{\prime \prime \prime}\right\}$, then we have 


$$
\begin{aligned}
& F_{1}=\left(1-\lambda c_{1}\right) \vec{\xi}_{1} \\
& F_{2}=\left(1-\lambda c_{1}\right) c_{1} \vec{\eta}_{1}-\left(1-\lambda c_{1}\right) \vec{\zeta}_{1} \\
& F_{3}=\frac{\left(1-\lambda c_{1}\right) c_{1}^{\prime}}{c_{1}^{2}+1} \vec{\eta}_{1}+\frac{\left(1-\lambda c_{1}\right) c_{1}^{\prime} c_{1}}{c_{1}^{2}+1} \vec{\zeta}_{1} .
\end{aligned}
$$

By [10], we have

$$
\kappa_{1}^{2}=b_{1}^{2}+c_{1}^{2}, b_{1}=1
$$

and

$$
\tau_{1}^{2}=-a_{1}+\frac{b_{1}^{\prime} c_{1}-b_{1} c_{1}^{\prime}}{b_{1}^{2}+c_{1}^{2}}, a_{1}=0
$$

By (8) and (9), we see that

$$
\tau_{1}=\frac{-c_{1}^{\prime}}{\kappa_{1}^{2}}
$$

By using (10) in $F_{3}$, we obtain

$$
F_{3}=-\left(1-\lambda c_{1}\right) \tau_{1} \vec{\eta}_{1}-\left(1-\lambda c_{1}\right) \tau_{1} \vec{\zeta}_{1}
$$

If we calculate $\kappa_{2}$ and $\tau_{2}$, then we have

$$
\kappa_{2}=\frac{\kappa_{1}}{\left|1-\lambda c_{1}\right|}
$$

and

$$
\tau_{2}=\frac{\tau_{1}}{\left|1-\lambda c_{1}\right|}
$$

Dividing by $\kappa_{2}$ to $\tau_{2}$, we obtain

$$
\frac{\kappa_{2}}{\tau_{2}}=\frac{\kappa_{1}}{\tau_{1}}
$$

We obtain the proof of the theorem from the last equation.

\section{References}

[1] Blaschke, W., Monatsheftefürmathematik und physik 19, pp:188-204, (1908).

[2] Blaschke, W., Differential Geometria, p:41, (1908).

[3] Ertem Kaya F (2010). Terquem and Joachimsthal Type Theorems in $E^{\mathrm{n}}[\mathrm{PhD}]$ Ankara University, Pages 72-78.

[4] Ertem Kaya F, Yaylı Y, Hacisalihoglu H., Harmonic Curvatures of a Strip in $E^{3}$, Commun. Fac. Sci. Univ. Ank. Series A1, Volume 59, Number 2, Pages 1-14 ISSN 1303-5991, (2010).

[5] Ertem Kaya F, Yaylı Y, Hacısalihoglu H., The Conical Helix Strip in $E^{3}$., Int. Journal of Pure and App. Mathematics, Volume 66, No(2), Pages:145-156, (2011).

[6] Hacisalihoglu H. H., Differential Geometry, Fasc. I, Science Faculty of Ankara University Publications, Besevler, Ankara (in Turkish).

[7] Hacisalihoglu H. H., Differential Geometry, Fasc. II, Science Faculty of Ankara University Publications, Besevler, Ankara (in Turkish).

[8] Hacisalihoglu H. H., On The Relations Between The Higher Curvatures Of A Curve and A Strip., Communications de la faculté des Sciences De Universitéd.AnkaraSerie A1, Tome 31, (1982).

[9] Hacisalihoglu H H., Lineer Algebra, Science Faculty of Ankara University Publications, Besevler, Ankara (in Turkish), (2008).

[10] Keles, S. Joachimsthal theorems for Manifolds [PHD], Firat University, Turkey, (1982).Communications de la faculté des Sciences De Universitéd.AnkaraSerie A1, Tome 31, (1982).

[11] Sabuncuoglu, A. and Hacisalihoglu H. H., Higher Curvatures of a Strip, Communications de la Faculté des Sciences De L.Universitéd.AnkaraSérie A1, Tome 24 , pp: 25-33, (1975).

[12] Scofield, P. D. "Curves of Constant Precession." Amer. Math. Monthly102, 531-537, 1995. 